\title{
Endolymphatic Sac Surgery for Meniere's Disease
}

\section{Stephen Wetmore}

\section{Source of support: Nil}

\section{Conflict of interest: None}

\section{INDICATIONS FOR THE SURGERY}

Endolymphatic sac surgery (ESS) is indicated for the treatment of patients, suffering with Meniere's disease (MD), who are recalcitrant to optimum medical therapy and in whom the disease is severely affecting their lifestyle. More specifically, ESS is used for the treatment of the major dizzy spells not for the adjunctive dizzy spells nor for the treatment of hearing loss, tinnitus or fullness in the ear or head. The indications for surgery depend, to some extent, on the patient's lifestyle. A retired individual may be willing to tolerate one or two major dizzy spells per week, but a truck driver would have difficulty tolerating one or two spells per month, especially, if there were no warning for the dizzy spells. Endolymphatic sac surgery has not proven effective for the treatment of hearing loss, tinnitus or fullness. Hearing loss often continues to get worse over time even in those individuals who have good or excellent control of their major dizzy spells. ${ }^{1,2}$

\section{SPECIFIC PREOPERATIVE EVALUATION}

A complete history and physical examination is performed and the patient's general medical condition is assessed. Usually, patients with MD who are between major dizzy spells will exhibit a normal Romberg test and a steady gait.

It is essential that the involved ear be correctly identified. Audiograms are evaluated to look for the characteristic low frequency sensorineural hearing loss. Some patients exhibit normal hearing between spells, especially early in the course of their disease; repeated testing is important in these individuals to look for fluctuating low frequency

\footnotetext{
Professor and Chair

Department of Otolaryngology, West Virginia University School of Medicine, Morgantown, West Virginia, USA
}

Corresponding Author: Stephen Wetmore, Professor and Chair Department of Otolaryngology, West Virginia University School of Medicine, Morgantown, West Virginia, USA sensorineural hearing loss. It is also important to assess the patient's auditory symptoms, looking for unilateral tinnitus, hearing loss and/or fullness. Electrocochleography (ECoG) may be helpful, if it shows elevated summating potentials in one ear; however, if that is the only evidence for identifying the involved ear, the surgeon would be reluctant to offer surgery to that patient because $\mathrm{ECoG}$ is not $100 \%$ reliable.

The author prefers to obtain a preoperative high resolution CT scan to assess the surgical anatomy, including the amount of air cell pneumatization and the location of the sigmoid sinus but the procedure can be safely performed without a scan.

\section{ANESTHETIC CONSIDERATIONS}

Endolymphatic sac surgery is usually performed under general anesthesia. To help with hemostasis, $1 \%$ lidocaine with 1:100,000 epinephrine can be injected along the planned postauricular incision. The anesthesiologist is asked not to paralyze the patient while the surgeon is operating so that facial nerve function can be monitored.

\section{SURGICAL STEPS}

The patient is positioned supine on the operating table with the involved ear turned up about $45^{\circ}$. A postauricular incision is made with a scalpel just a few millimeters posterior to the postauricular crease from the superior attachment of the pinna to the mastoid tip. Dissection is carried anteriorly in the plane just superficial to the temporalis fascia and the sternocleidomastoid fascia. In the superior part of the surgical field, the dissection is carried toward the zygomatic root, in the inferior part, the dissection is carried to the level of the external auditory canal being careful not to enter that structure. Dissection is carried more anteriorly in the superior part of the field than in the inferior portion, similar to what is done during a standard mastoidectomy. A 'T-shaped' incision is made through the muscle and periosteum to the bone of the mastoid cortex. The anterior limb of the ' $T$ ' extends to the anterior aspect of the dissection in the zygomatic root. Periosteal elevators are used to expose the cortex and the bony external auditory canal (EAC) is identified. If a large spine of Henle is present, it can be drilled off in order to be sure of the location of 
the EAC. Two self-retaining retractors are inserted to help visualize the mastoid cortex.

The operating microscope is brought into the surgical field and a complete mastoidectomy is performed using cutting burrs. The antrum is entered and the lateral semicircular canal is identified. Drilling is continued into the zygomatic root to identify the incus. The remainder of the zygomatic root need not to be opened. The remainder of the air cells in the mastoid are exenterated, identifying the tegmen mastoideum, the sinodural angle, the sigmoid sinus and the mastoid tip (Fig. 1). The air cells are also removed from the posterior EAC but the posterior EAC is kept intact.

The bone covering the sigmoid sinus is thinned using diamond burrs (Fig. 2) The endolymphatic sac is located in the region of the cerebellar plate of bone that extends from the anterior aspect of the sigmoid sinus to the area of the posterior semicircular canal (PSC). Sometimes the sigmoid sinus bulges anteriorly making it difficult to visualize the cerebellar plate of bone. In that circumstance, it may be helpful to completely remove the bone overlying the sigmoid sinus so that the sinus can be gently depressed in order to visualize the region where the endolymphatic sac should lie. The bone of the cerebellar plate is removed using diamond burrs. Dura in this region is exposed widely extending superiorly almost to the tegmen and inferiorly and anteriorly to the mastoid tip and retrofacial areas, respectively (Figs 3A and $\mathrm{B})$. Dissection is done from a posterior to an anterior direction in order to identify the PSC. In some patients, the PSC is easy to identify, but in other patients identification may be more difficult. In most patients, small air cells are present in the acute angle between the cerebellar plate and the PSC (Fig. 4). Carefully removing these cells helps the surgeon to identify the PSC.

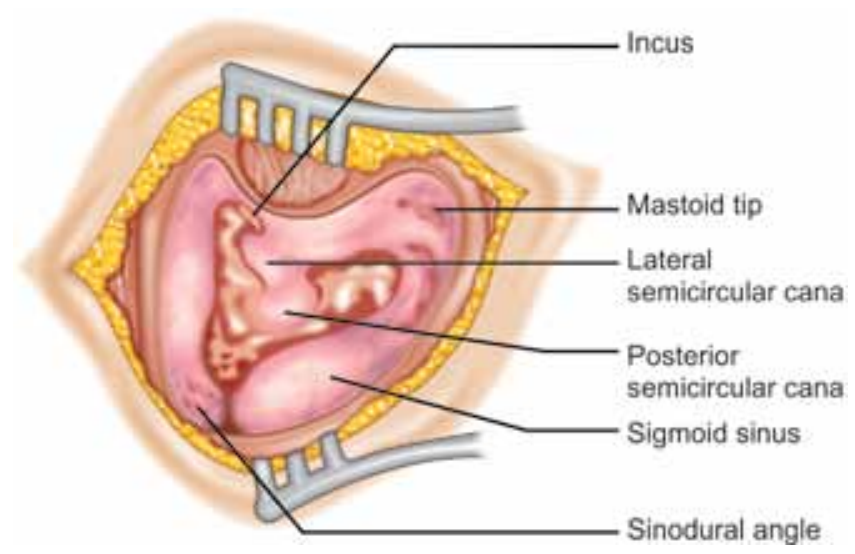

Fig. 1: A complete mastoidectomy has been performed in this right ear displaying the incus, the lateral and posterior semicircular canals and the sigmoid sinus
The endolymphatic sac and duct are usually located at, or inferior to, Donaldson's line, the imaginary line extending from the lateral semicircular canal and bisecting the PSC (Figs 3A and B). The endolymphatic sac is a thickening of the dura and appears to be whiter than the more blue color of the remainder of the dura. Widely exposing the dura will help to distinguish this color difference. Occasionally, this difference in color will not be easy to see. The endolymphatic duct extends anteriorly, deep to the PSC.

Most surgeons will open the sac and place a prosthesis in the lumen to theoretically drain the sac into the mastoid cavity. If the patient has small blood vessels crossing in the area where the author wishes to open the sac, he will use a bipolar cautery to coagulate the vessels. The author uses a small right angle pick to dissect the superficial layer of dura to make a small opening from the PSC toward the sigmoid sinus (Fig. 5). Then, he uses a blunt ended instrument, such as a Rosen needle to identify a lumen and to identify the endolymphatic duct. A variety of prostheses have been devised to place in the lumen of the sac. The author uses a tiny arrow-shaped piece of 0.010 inch $(0.254 \mathrm{~mm})$ silastic sheeting (PharmElast) that is hand carved with small instruments. The arrowhead is placed into the lumen of the sac with the tail extending into the mastoid cavity (Fig. 6).

Published reports indicate that whether the sac is opened or merely decompressed from the overlying bone makes no difference in the ultimate success in relieving major dizzy spells. ${ }^{3}$ Recently, when the author has had difficulty identifying the sac or when he is operating on an elderly patient with thin dura, the author has elected to decompress the sac and not attempted to place a prosthesis in the lumen.

The surgical site is sutured in layers and a mastoid dressing is applied and left in place for one day.

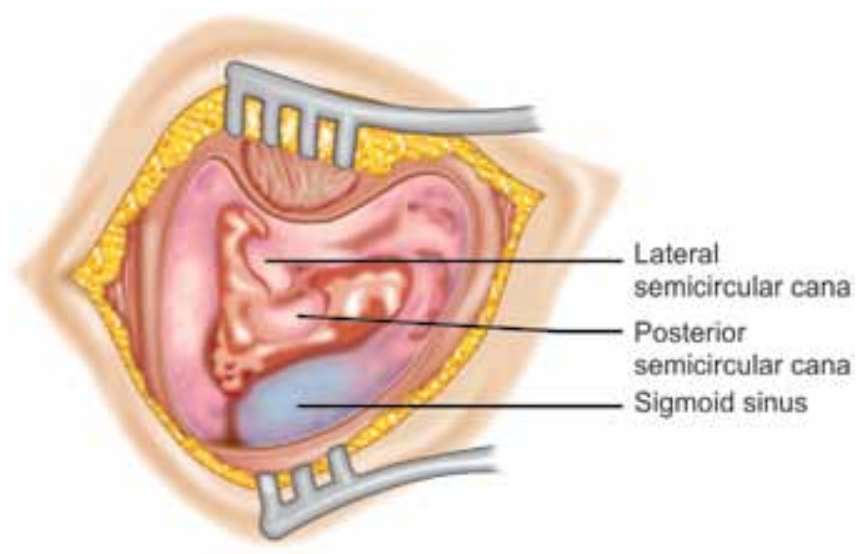

Fig. 2: The sigmoid sinus has been skeletonized showing the blue color of the sinus visible through the bone 

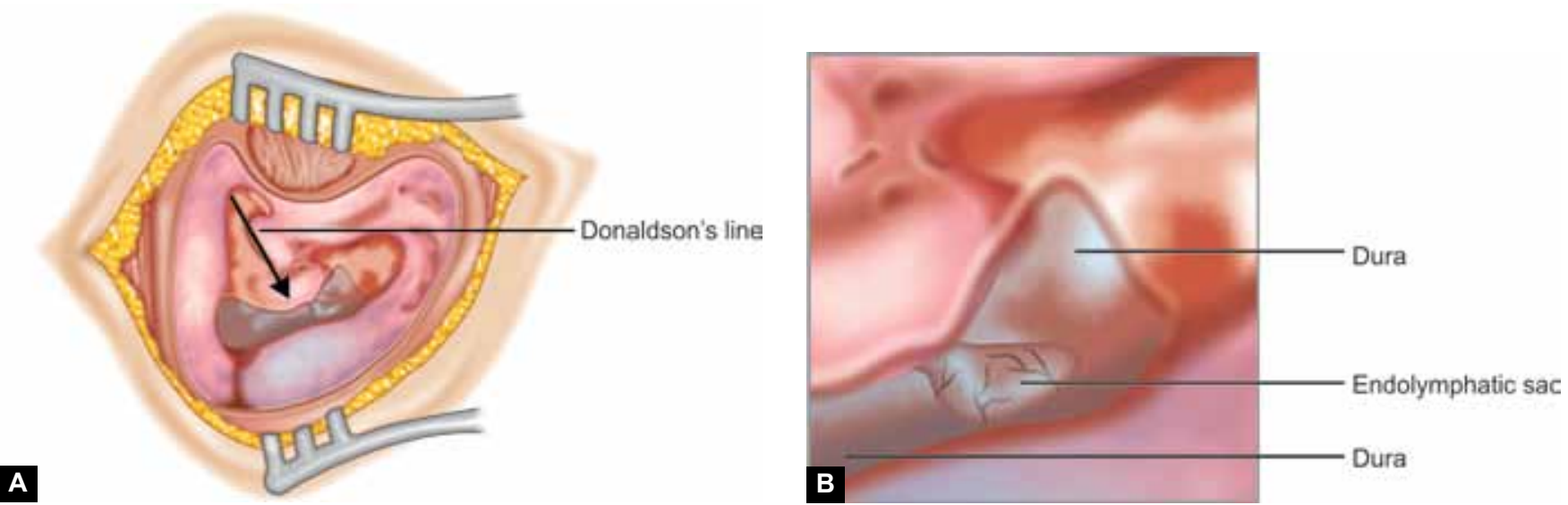

Figs 3A and B: $(A)$ The bone overlying the posterior fossa dura has been widely removed in order to identify the white color of the endolymphatic sac compared with the blue color of the rest of the dura. The sac is usually located at or inferior to Donaldson's line (arrow) and (B) Higher magnification of $A$

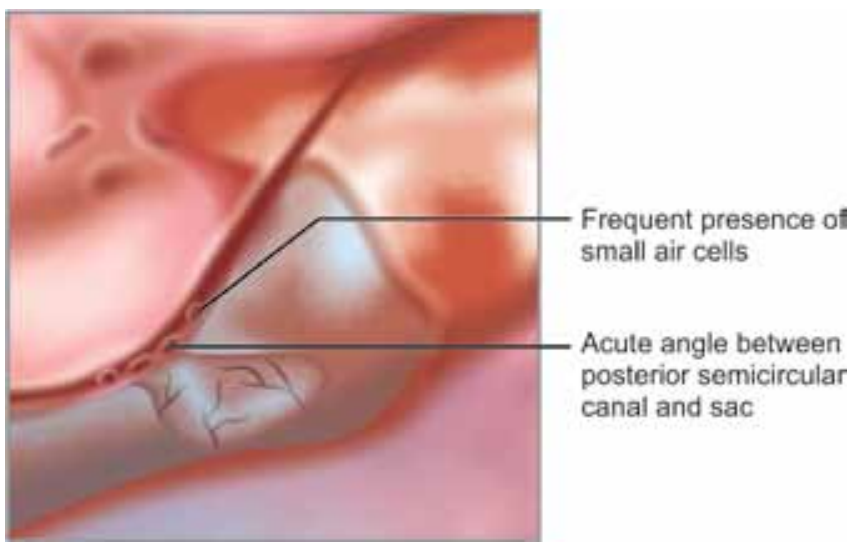

Fig. 4: A few small air cells are usually located in the sharp angle between the posterior semicircular canal and the posterior fossa dura and sac

\section{NEW TECHNIQUES IN THE SURGERY}

In the past year or two, the author has been more conservative regarding opening the sac. If the patient is elderly and/or the author has difficulty determining the location of the sac, he will often elect to just decompress the sac.

\section{COMPLICATIONS}

A cerebrospinal fluid (CSF) leak can occur if the surgeon dissects through the full thickness of the dura while trying to find the lumen of the endolymphatic sac. Even a pinpoint opening in the dura may result in a fairly copious leakage of CSF. After waiting for the fluid to subside in amount, the surgeon can try to place a tiny piece of fascia or fat into the hole. Suturing the dura for a tiny leak often does not stop the leak but may make the hole larger. Another option is obtaining a fat graft from the abdominal wall and filling the cavity with the graft, often in conjunction with the use of fibrin glue. In the case of a CSF leak, placement of prosthesis into the lumen of the sac is abandoned.

Other potential complications include drilling into the posterior semicircular canal, which can result in chronic

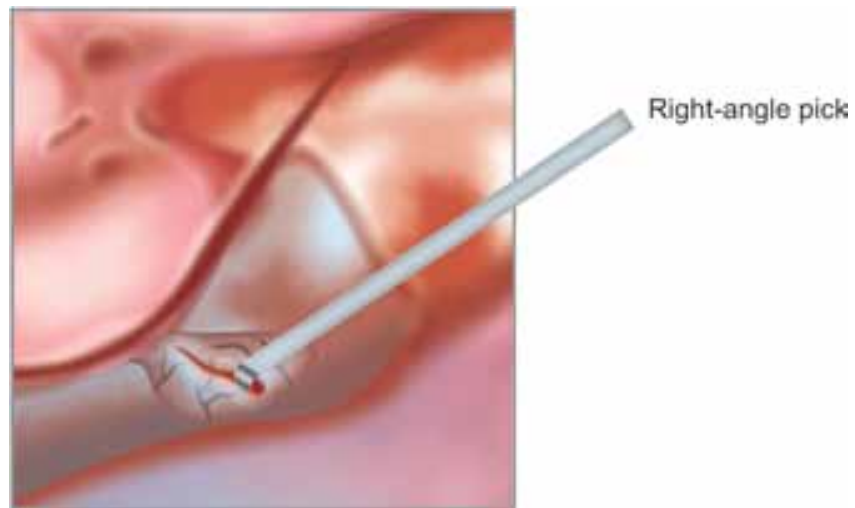

Fig. 5: An incision is made carefully through the lateral portion of the sac using a small right-angled pick

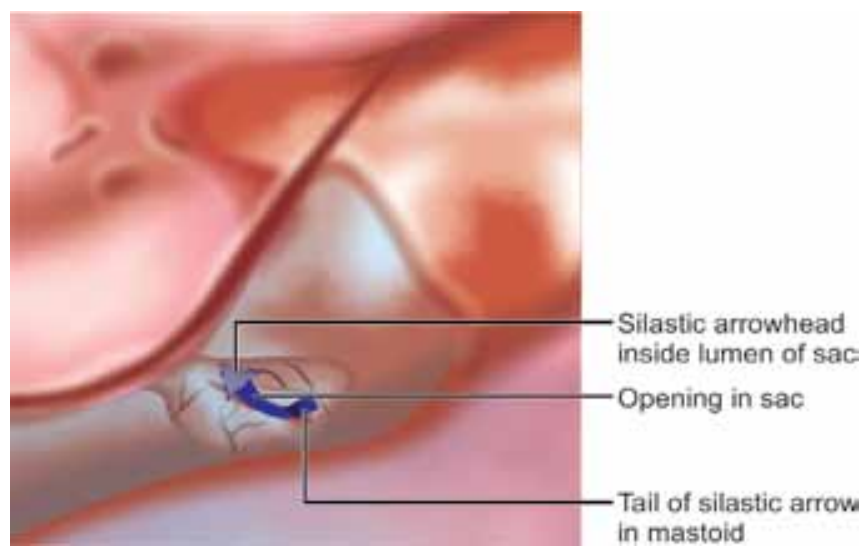

Fig. 6: A silastic prosthesis made in the shape of an arrow is placed into the lumen of the sac with tail of the arrow extending into the mastoid cavity

unsteadiness and a profound hearing loss, facial paralysis from drilling into the facial nerve and hemorrhage due to injury to the sigmoid sinus. Most bleeding from the sigmoid sinus can be controlled by placing a Gelfoam patch over the damaged area of the sigmoid sinus and holding the patch in place for a few minutes with a cottonoid sponge. After gently removing the cottonoid sponge, the Gelfoam patch is left in place. 


\section{SPECIAL INSTRUMENTS USED FOR THE SURGERY}

A delicate right angled pick is helpful to incise the lateral surface of the dura overlying the endolymphatic sac and a blunt needle, such as the Rosen needle, is useful to identify the lumen of the sac. If prominent vessels are noted overlying the sac, cauterizing these vessels with a delicate bipolar cautery is useful.

\section{OTHER TREATMENT OPTIONS AVAILABLE FOR THE SAME CONDITION}

A variety of other options are available for the treatment of Meniere's disease that is recalcitrant to medical management. These options include intratympanic injection of gentamicin, intratympanic injection of corticosteroids, the Meniett device, vestibular nerve section and labyrinthectomy.

Intratympanic injection of gentamicin is a popular treatment option that can be performed in the outpatient clinic. After anesthetizing the tympanic membrane, a dose of gentamicin is injected into the middle ear while the patient lies still for approximately 30 minutes. A variety of protocols regarding dose and frequency of instillation have been published. Most published studies have shown that the majority of patients obtain a good result in regard to the frequency and severity of major dizzy spells. ${ }^{4,5}$ The main problem with this treatment modality is the relatively high incidence of hearing loss that has been reported.

Intratympanic injection of a corticosteroid preparation, primarily prednisolone or dexamethasone, has been reported to help alleviate the major dizzy spells associated with MD. The advantage of this product over gentamicin is the virtual absence of hearing loss from this drug; the disadvantage is that corticosteroid preparations appear to be considerably less efficacious than gentamicin in regards to treating the major dizzy spells. ${ }^{6}$

The Meniett device provides pressure to the inner ear in the hope of alleviating the major dizzy spells. The device is applied to the ear canal of the affected ear for 5 minutes three times a day after first placing a ventilating tube in the tympanic membrane. Although, some reports show good results using the device, it has not become universally popular. ${ }^{7,8}$

Cutting the vestibular portion of cranial nerve eight results in alleviation of the major dizzy spells in a fairly high percentage of patients suffering from MD. The procedure can be performed through a middle fossa, retrolabyrinthine or retrosigmoid approach. The main disadvantages are the greater overall risks of an intracranial procedure, the risk of total hearing loss, and the temporary dizziness due to ablation of the vestibular portion of the eighth cranial nerve.

Labyrinthectomy results in a high degree of success in alleviating major dizzy spells but does result in profound hearing loss in the operated ear and the temporary dizziness associated with ablation of vestibular function in one ear.

Revision sac surgery is an option for someone who initially experienced good results after sac surgery but who develops recurrence of symptoms. The definition of 'good results' implies that the individual exhibited complete or nearly complete resolution of the major dizzy spells for 6 months following the initial sac surgery. Studies have showed that patients who have done well for 2 years or more prior to recurrence of major dizzy spells do better after revision sac surgery than those who recur within 2 years of their initial sac procedure. ${ }^{1,9}$

\section{REFERENCES}

1. Wetmore SJ. Endolymphatic sac surgery for Meniere's disease: long-term results after primary and revision surgery. Arch Otolaryngol Head Neck Surg 2008;134(11):1144-1148.

2. Sun GH, Leung R, Samy RN, et al. Analysis of hearing preservation after endolymphatic mastoid sac surgery for Meniere's disease. Laryngoscope 2010;120(3):591-597.

3. Brinson GM, Chen DA, Arriaga MA. Endolymphatic mastoid shunt versus endolymphatic sac decompression for Meniere's disease. Otolaryngol Head Neck Surg 2007;136(3):415-421.

4. Chia SH, Gamst AC, Anderson JP, et al. Intratympanic gentimicin therapy for Meniere's disease: a meta-analysis. Otol Neurotol 2004;25(4):544-552.

5. Cohen-Kerem R, Kisilevsky V, Einarson TR, et al. Intratympanic gentimicin for Meniere's disease: a meta-analysis. Laryngoscope 2004;114(12):2085-2091.

6. Doyle KJ, Bauch C, Battista R, et al. Intratympanic steroid treatment: a review. Otol Neurotol 2004;25(6):1034-1039.

7. Densert B, Sass K. Control of symptoms in patients with Meniere's disease using middle ear pressure applications: two years follow-up. Acta Otolaryngol 2001;121(5):616-621.

8. Gates GA, Verrall A, Green JD, et al. Meniett clinical trial: long-term follow-up. Arch Otolaryngol Head Neck Surg 2006; 132(12):1311-1316.

9. Huang TS, Lin CC. Revision endolymphatic sac surgery for recurrent Meniere's disease. Acta Otolaryngol Suppl 1991; 485:131-144. 